\title{
WFI electronics and on-board data processing
}

Plattner, Markus; Albrecht, Sebastian; Bayer, Jörg; Brandt, Søren; Drumm, Paul; Hälker, Olaf; Kerschbaum, Franz; Koch, Anna; Kuvvetli, Irfan; Meidinger, Norbert

Total number of authors:

18

Published in:

Proceedings of Space Telescopes and Instrumentation 2016: Ultraviolet to Gamma Ray

Link to article, DOI:

$10.1117 / 12.2235375$

Publication date:

2016

Document Version

Publisher's PDF, also known as Version of record

Link back to DTU Orbit

Citation (APA):

Plattner, M., Albrecht, S., Bayer, J., Brandt, S., Drumm, P., Hälker, O., Kerschbaum, F., Koch, A., Kuvvetli, I., Meidinger, N., Ott, S., Ottensamer, R., Reiffers, J., Schanz, T., Skup, K., Steller, M., Tenzer, C., \& Thomas, C. (2016). WFI electronics and on-board data processing. In J-W. A. den Herder, T. Takahashi, \& M. Bautz (Eds.), Proceedings of Space Telescopes and Instrumentation 2016: Ultraviolet to Gamma Ray (Vol. 9905). [99052D] SPIE - International Society for Optical Engineering. https://doi.org/10.1117/12.2235375

\section{General rights}

Copyright and moral rights for the publications made accessible in the public portal are retained by the authors and/or other copyright owners and it is a condition of accessing publications that users recognise and abide by the legal requirements associated with these rights.

- Users may download and print one copy of any publication from the public portal for the purpose of private study or research.

- You may not further distribute the material or use it for any profit-making activity or commercial gain

- You may freely distribute the URL identifying the publication in the public portal 


\title{
WFI Electronics and On-Board Data Processing
}

\author{
Markus Plattner $^{\text {la }}$, Sebastian Albrecht ${ }^{\mathrm{a}}$, Jörg Bayer ${ }^{\mathrm{b}}$, Soren Brandt ${ }^{\mathrm{f}}$, Paul Drumm ${ }^{\mathrm{g}}$, Olaf Hälker ${ }^{\mathrm{a}}$, Franz Kerschbaum ${ }^{\mathrm{c}}$ \\ Anna Koch ${ }^{\mathrm{a}}$, Irfan Kuvvetli ${ }^{\mathrm{f}}$, Norbert Meidinger ${ }^{\mathrm{a}}$, Sabine Ott ${ }^{\mathrm{a}}$, Roland Ottensamer ${ }^{\mathrm{c}}$, Jonas Reiffers ${ }^{\mathrm{a}}$, Thomas Schanz ${ }^{\mathrm{b}}$, \\ Konrad Skup ${ }^{\mathrm{e}}$, Manfred Steller ${ }^{\mathrm{d}}$, Chris Tenzer ${ }^{\mathrm{b}}$, Chris Thomas ${ }^{\mathrm{g}}$, \\ ${ }^{a}$ Max Planck Institute for Extraterrestrial Physics, Gießenbachstr. 1, 85748 Garching, Germany \\ ${ }^{\mathrm{b}}$ University Tübingen, Institut für Astronomie und Astrophysik, Sand 1, 72076 Tübingen, Germany \\ ${ }^{\mathrm{c}}$ University Vienna, Institut für Astrophysik, Türkenschanzstr. 17, 1180 Wien, Austria \\ ${ }^{\mathrm{d}}$ Austrian Academy of Science, Space Research Institute, Schmiedlstraße 6, 8042 Graz, Austria \\ ${ }^{\mathrm{e}}$ Polish Academy of Science, Space Research Center, Bartycka 18A, 00-716 Warsaw, Poland \\ ${ }^{\mathrm{f}}$ Technical University of Denmark, National Space Institute, Elektrovej, 2800 Kgs. Lyngby, Denmark \\ ${ }^{g}$ University of Leicester, Department of Physics and Astronomy, University Road, Leicester LE1 7RH, UK
}

\begin{abstract}
The Wide Field Imager is one of two instruments on-board the future ATHENA X-ray observatory. Its main scientific objective is to perform a sky survey in the energy range of $0.2 \mathrm{keV}$ up to $15 \mathrm{keV}$ with an end-of-life spectral resolution (FWHM) better than $170 \mathrm{eV}$ (at $7 \mathrm{keV}$ ) and a frame rate of at least $200 \mathrm{~Hz}$. The field of view will be 40 arcmin squared wherefore a focal plane array with 4 large sensors each with a size of 512 times 512 pixels will be developed. Additionally, a fast detector with a size of 64 times 64 pixels and a frame rate of $12.5 \mathrm{kHz}$ will be implemented in order to enhance the instrument with high count rate detection of bright sources.

The data processing electronics within the WFI instrument is distributed over several subsystems: DEPFET sensors sensitive in the $x$-ray energy regime and front-end electronics are located inside the Camera Head. Data pre-processing inside the Detector Electronics will be performed in an FPGA-based frame-processor. FPGA external memory will be used to store offset and noise maps wherefore memory controllers have to be developed. Fast read and write access to the maps combined with robustness against radiation damage (e.g. bit-flips) has to be ensured by the frame-processor design.
\end{abstract}

Keywords: Athena, WFI, Wide Field Imager, data pre-processing, frame processor

\section{INTRODUCTION}

\subsection{WFI Specification}

The Wide Field Imager (WFI) is one of two instrument on-board ESA's next generation X-ray observatory Athena. The main scientific objectives [1], that drive WFI electronics design are

- energy range from $0.2 \mathrm{keV}$ up to $15 \mathrm{keV}$,

- energy resolution according to the requirements, e.g. $\operatorname{FWHM}(1 \mathrm{keV}) \leq 80 \mathrm{eV}$,

- energy knowledge for WFI calibration of $10 \mathrm{eV}$,

- with a field of view of 40 arc min squared at a frame period of $\leq 5 \mathrm{~ms}$,

- time resolution of $\leq 80 \mu$ s per frame for observation of bright point sources,

${ }^{1}$ plm@mpe.mpg.de, phone: +49 8930000 3824, www.mpe.mpg.de

Space Telescopes and Instrumentation 2016: Ultraviolet to Gamma Ray, edited by

Jan-Willem A. den Herder, Tadayuki Takahashi, Marshall Bautz, Proc. of SPIE Vol. 9905, 99052D

(C) 2016 SPIE · CCC code: 0277-786X/16/\$18 · doi: 10.1117/12.2235375 
WFI will be subjected to various environmental conditions throughout its life cycle. The most critical ones with regard to impact on design are launch, orbit transfer and in-orbit operation. For WFI electronics design, the radiation environment is of particular interest. According to ESA specification, the baseline orbit for ATHENA will be around Lagrange Point 2. Under the assumption of an extended mission lifetime (10 years) WFI will be subjected to a total ionizing dose (TID) at the level of $8.7 \mathrm{krad}(\mathrm{Si})$ and a non-ionizing energy loss (NIEL) of $1.1 \times 10^{8} \mathrm{MeV} / \mathrm{g}$ (Si) taking into account $5 \mathrm{~mm}$ Aluminum-equivalent shielding [2].

With regard to electronics within the subsystems DE and ICPU, these radiation loads have to be considered as one of the boundary conditions for starting a conceptual design. Radiation design margins according to ECSS standards have to be applied but additional shielding due to FPM, radiators and other payload instruments have not been taken into account up to now. A proton-shield with an Aluminum equivalent shielding of approximately $4 \mathrm{~cm}$ has been included in the $\mathrm{CH}$ design in order to minimize NIEL damage of the DEPFET sensors (see right hand side of Figure 1). Thereby, TID and NIEL for DEPFET sensors and front-end electronics is reduced significantly by roughly an order of magnitude.

The Athena mission is scheduled for a launch in 2028. In the current phase A, technology development is performed in order to reach a certain technical maturity of all components that are critical with regard to an operation in space. Within WFI, the critical electronics are the DEPFET-based detectors and the real-time signal pre-processing.

\subsection{WFI Hardware Architecture}

The WFI payload instruments will be accommodated in the Focal Plane Module (FPM) of the Athena spacecraft. An instrument Primary Structure consists of a baseplate and four bi-pods. Together with the WFI subsystems Filter Wheel (FW), Camera Head (CH) and Detector Electronics (DE) the so-called WFI Focal Plane Assembly (FPA) is integrated as shown in Figure 1. In addition to the WFI FPA, a further subsystem called Instrument Control and Power-distribution Units (ICPU) is needed. Because of FPM center-of-gravity balancing, ICPUs will be mounted at a different place inside FPM some distance away from the WFI FPA.
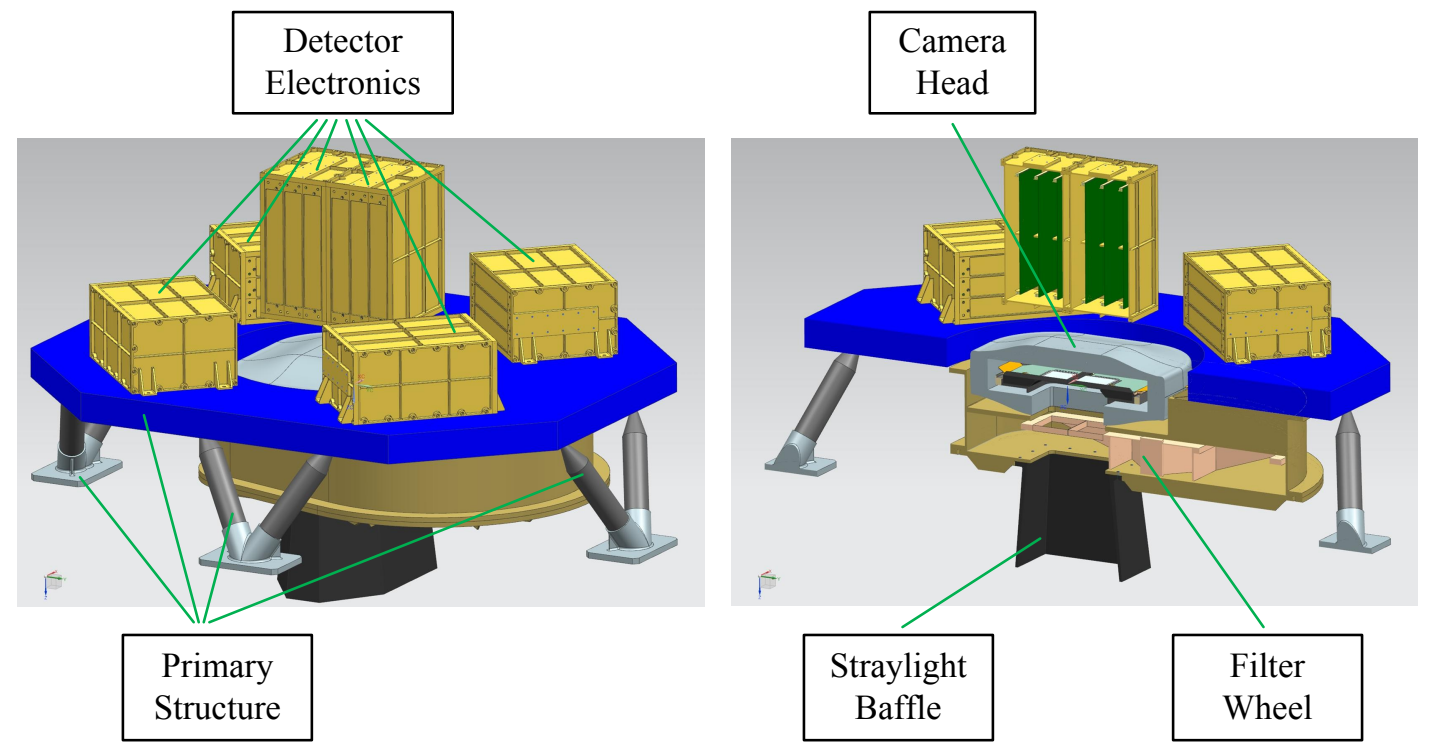

Figure 1. WFI FPA 3-dimensional concept drawing and cross-sectional view.

WFI CH, DEs and ICPUs are power consuming subsystems that have to be cooled. Heat pipe interfaces for each subsystem are provided in order to thermally connect WFI to radiators mounted on the FPM.

The WFI instrument is based on DEPFET active-pixel sensors. One sensor combined with its detector PCB that holds the front-end electronics (FEE) and its mechanical support structure is called a detector. A Large Detector (LD) consisting of four quadrants and one Fast Detector (FD) are implemented in WFI. The four LD quadrants are configured in an array in 
order to provide the 40x40 $\mathrm{arcmin}^{2}$ field of view as shown in Figure 2. The FD is mounted defocused which improves its high-count rate capability. The four LD quadrants and the FD have a size of 512 x 512 and 64 x 64 pixels respectively.

All WFI detectors are surrounded by graded- $Z$ and proton shielding in order to minimize instrumental background and radiation damage. Each detector PCB has rigid and flexible parts: FEE are mounted at the rigid detector boards next to the sensors in order to enable wire bonding. The flexible parts are used as connections of the detectors inside the $\mathrm{CH}$ to the DEs that are part of the WFI FPA. In order to achieve real-time processing in the DE subsystem and to increase instrument reliability, each LD quadrant is connected to one of four dedicated DE subsystem units. The FD is read-out in two halves and therefore connected to two DE subsystem units.

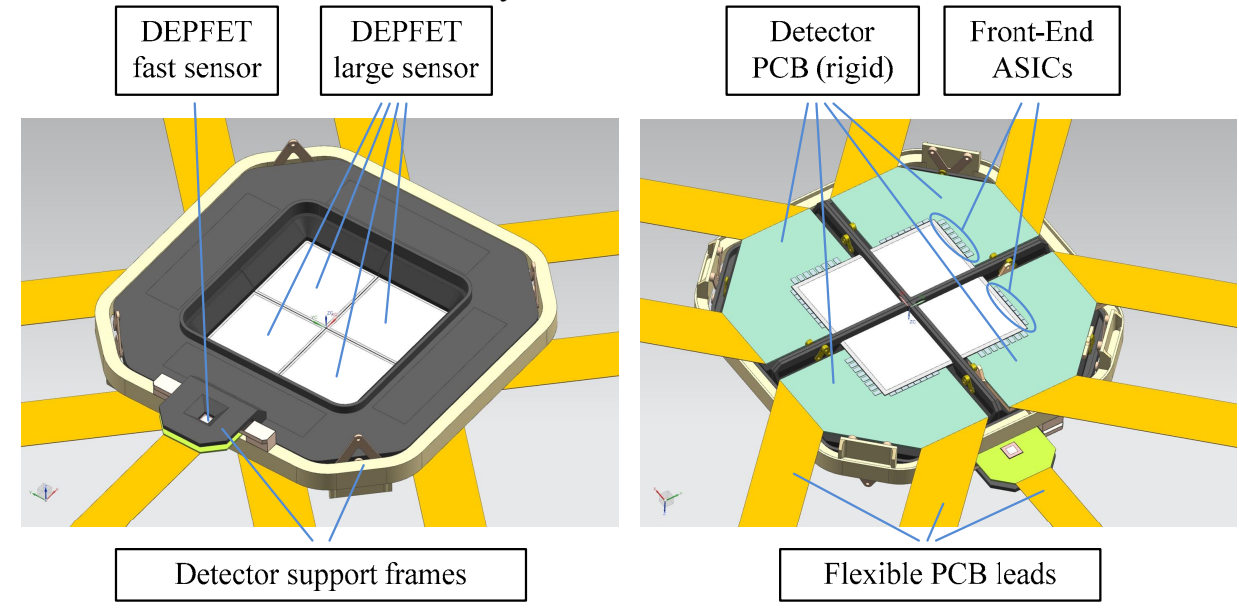

Figure 2. WFI detectors (inside Camera Head). Left: Front view, right: back view.

\section{WFI FUNCTIONALITY AND PERFORMANCE}

\subsection{WFI Concept of Operation}

The WFI operational concept and its data acquisition and processing concept is illustrated in Figure 3. Photons focused to WFI by the Athena mirror system pass the filter wheel. An optical and UV filter shall block UV and visible light photons but not the X-ray photons in the WFI energy range. X-ray photons that hit the DEPFET sensor generate signal charges in 1,2, 3 or 4 pixels, depending on the point of incidence. Charges proportional to the X-ray photon energy are generated and stored in the up to four DEPFET pixel(s).

The detectors are operated in rolling shutter mode: The entire sensor is biased and active at all measurement times. Switcher-A ASICs are the main components of the Control Front-end Electronics (CFE). One Switcher-A is able to control 64 sensor rows wherefore a number of eight Switcher-A devices are implemented in one LD quadrant. The CFE of each detector is controlled by the corresponding DE such that the sensor is read-out row-by-row. The FD is read-out in two halves wherefore two Switcher-A devices are implemented and two rows (one per half of the fast sensor) are readout simultaneously. 


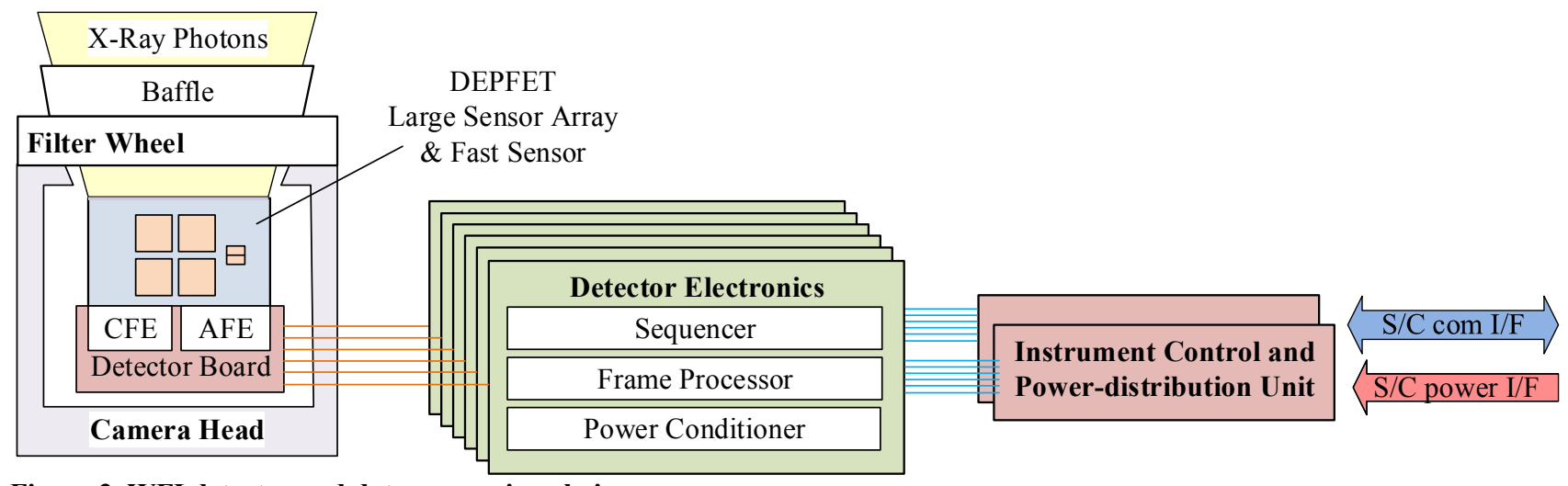

Figure 3. WFI detector and data processing chain.

The signals are further amplified and shaped in the Analog Front-end Electronics (AFE). The main components of AFE are the Veritas-2 ASICs. One Veritas-2 is able to read-out 64 sensor columns wherefore a number of eight Veritas-2 devices are implemented in one LD quadrant. Since the FD is read-out in two halves, two Veritas-2 devices are implemented in the FD to read-out two rows (one per half of the fast sensor) simultaneously. All pixels of one active sensor row (activated by CFE) are read-out in parallel. All measurement signals are stored as charges inside the ASICs and clocked-out to one serial output buffer per Veritas-2 device. The analog differential output signals of all Veritas-2 output channels are transferred to the corresponding DEs.

One DE for a LD quadrant provides a number of eight analog input channels required to digitize all eight Veritas-2 output channels of the corresponding detector. The two DEs corresponding to the FD have one analog input channel each. The science data streams are combined detector-wise in an FPGA-based Frame Processor (FP) which performs scientific data pre-processing (see section 2.2).

Furthermore, the DE also contains a Sequencer module which is required for controlling and setting parameters of CFE and AFE. The Power Conditioner module is required for biasing the detector. The ICPU merges the data streams of all DEs and performs data compression (see section 2.3).

\subsection{Frame Processing}

The downlink rate of Athena observatory is limited to approximately $75 \mathrm{Gbit} / \mathrm{day}$. This is the main driver for on-board data pre-processing performed by the DEs. The high raw-data rates as output of the $\mathrm{CH}$ include all pixels of a detector. First, the DE digitizes all analog data. Second, data pre-processing is performed in the FP:

- Pixel-wise offset and common mode correction.

○ 200 dark frames (FW closed) are recorded before starting an observation. The baseline level of each pixel is calculated as average over the dark frames and stored as offset map in the FP memory.

- For each frame the baseline fluctuation of all pixels, the so-called common mode, of each row is calculated.

$\circ$ Each measurement frame is processed by pixel-wise subtraction of offset and common-mode. Thereby all pixel values are normalized.

- Event filtering and background reduction.

- Digitized pixel values are compared with the lower threshold in order to eliminate invalid events mainly due to noise that are outside the WFI energy range. Applying the lower threshold significantly reduces the data rate since all pixels that have not been hit by an X-ray photon are eliminated.

$\circ$ Applying the upper threshold filters high energy events above $15 \mathrm{keV}$. Instrumental background can be reduced erasing all measurement data of one quadrant if an energy deposition by such a high energy particle occurs (see [3]). Although the signal values are lost by this procedure, simulations revealed 
that background reduction in the order of $40 \%$ can be achieved which is an important gain in performance when observing faint sources.

- Event recombination.

A valid X-ray photon that hits a detector can spread its signal charge across up to four pixels. Invalid events that are due to particle hits usually affect more than four pixels. Identification of invalid pixel pattern allows for correction. The invalid events are erased from the event list which reduces the data rate in addition.

Figure 4 shows the calculated data rates inside the WFI instrument.

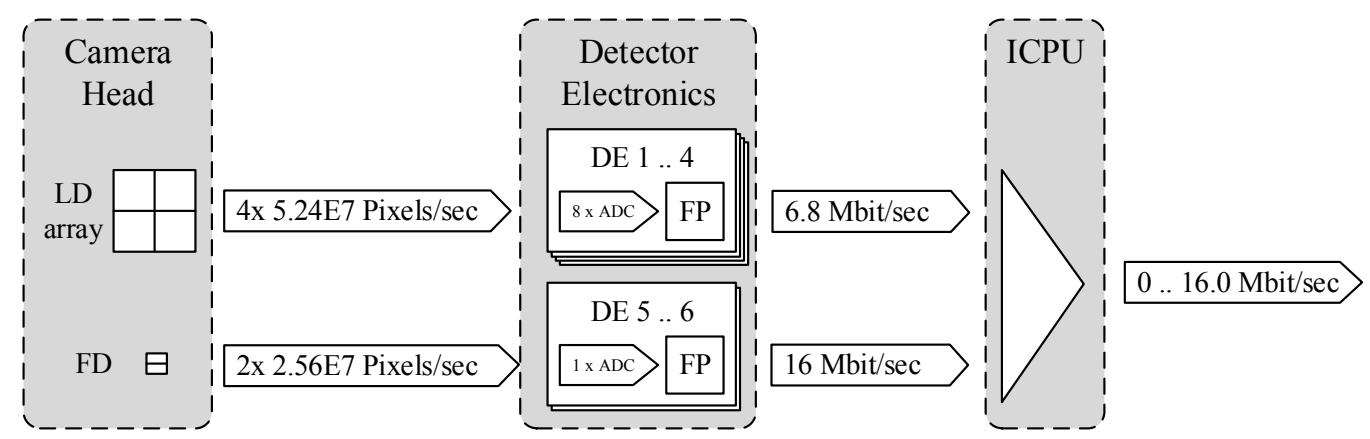

Figure 4. WFI data rates per subsystem

The DE output is an event list, i.e. every valid event due to an incoming x-ray photon in the energy range of interest is listed by its parameters photon energy and incidence location. One event list per frame is generated and sent to the ICPU together with a frame timestamp. The relevant science requirements for WFI [1] and the corresponding data rate calculations are summarized in Table 1.

Table 1. WFI requirements and data rate calculation.

\begin{tabular}{|l|r|r|r|r|r|r|r|r|r|r|} 
& $\begin{array}{l}\text { Frames per } \\
\text { second [1/s] }\end{array}$ & $\begin{array}{l}\text { CH read-out } \\
\text { rate [Pixel/s] }\end{array}$ & $\begin{array}{l}\text { px per event } \\
\text { (average) }\end{array}$ & $\begin{array}{l}\text { ADC } \\
\text { bits }\end{array}$ & $\begin{array}{l}\text { Position } \\
\text { bits }\end{array}$ & $\begin{array}{l}\text { FP internal raw } \\
\text { data rate [bit/s] }\end{array}$ & $\begin{array}{l}\text { Max. no. of } \\
\text { events per sec. }\end{array}$ & $\begin{array}{l}\text { TS bits per } \\
\text { frame }\end{array}$ & $\begin{array}{l}\text { DE output data } \\
\text { rate [bit/s] }\end{array}$ \\
\hline LDA & 200 & $2.10 \mathrm{E}+08$ & 2 & 14 & 20 & $1.78 \mathrm{E}+09$ & 100000 & 25 & $6.81 \mathrm{E}+06$ \\
\hline FD & 12500 & $5.12 \mathrm{E}+07$ & 2 & 14 & 12 & $6.66 \mathrm{E}+08$ & 300000 & 31 & $1.60 \mathrm{E}+07$ \\
\hline
\end{tabular}

The frame rates of $200 \mathrm{~Hz}(\mathrm{LD})$ and $12.5 \mathrm{kHz}(\mathrm{FD})$ result in analog $\mathrm{CH}$ output data rates of $2.10 \cdot 10^{8}$ and $5.12 \cdot 10^{7}$ pixels per second respectively. The maximum number of events per second depends on the WFI observation mode. When sources are observed during e.g. sky survey with the LD, a maximum of $1 \cdot 10^{5}$ events per second is assumed. For observation of bright sources with intensities up to 2.5 Crab using the FD, a maximum of $3 \cdot 10^{5}$ events per second occurs. One valid event can affect up to four pixels, two pixels on average are assumed.

In order to achieve the energy resolution requirement for WFI calibration $(10 \mathrm{eV})$, the ADC has to provide an effective number of bits (ENOB) of at least 11. Typical space qualified ADCs with 14 bit of resolution provide an ENOB of more than 11 bits. In addition to the 14 bit of energy resolution 20 and 12 bits of pixel position information is stored per event detected by the LDA and the FD respectively. Observations take a time up to $1 \cdot 10^{5}$ seconds wherefore 25 resp. 31 timestamp bits per frame have to be included to the total data rate.

Comparison of the equivalent raw-data rate from the $\mathrm{CH}$ and the pre-processed data as output of the DEs (see Figure 4) reveals data reduction factors of 430 and 45 for LDA and FD respectively. These theoretical calculation results are only achievable, if real-time data pre-processing in Frame Processor (FP) is working error-free. In reality, not all invalid events might by detected as such. Together with noise events exceeding the lower event threshold, the maximum data rates can be higher than calculated.

Nevertheless, these estimations show that its worth to put some effort in the optimization of the FP algorithms in order to maximize the science return of WFI. Section 3.2 introduces the WFI emulator that is a helpful tool for FP optimization. 


\subsection{Instrument Control and Power-distribution Unit}

All electrical I/F connections between WFI and the spacecraft (SC) are situated at the ICPU as shown in Figure 5. The ICPU is responsible for controlling the instrument and distributing SC power to all subsystems. The ICPU thereby is the power and communication interface between WFI and the SC.

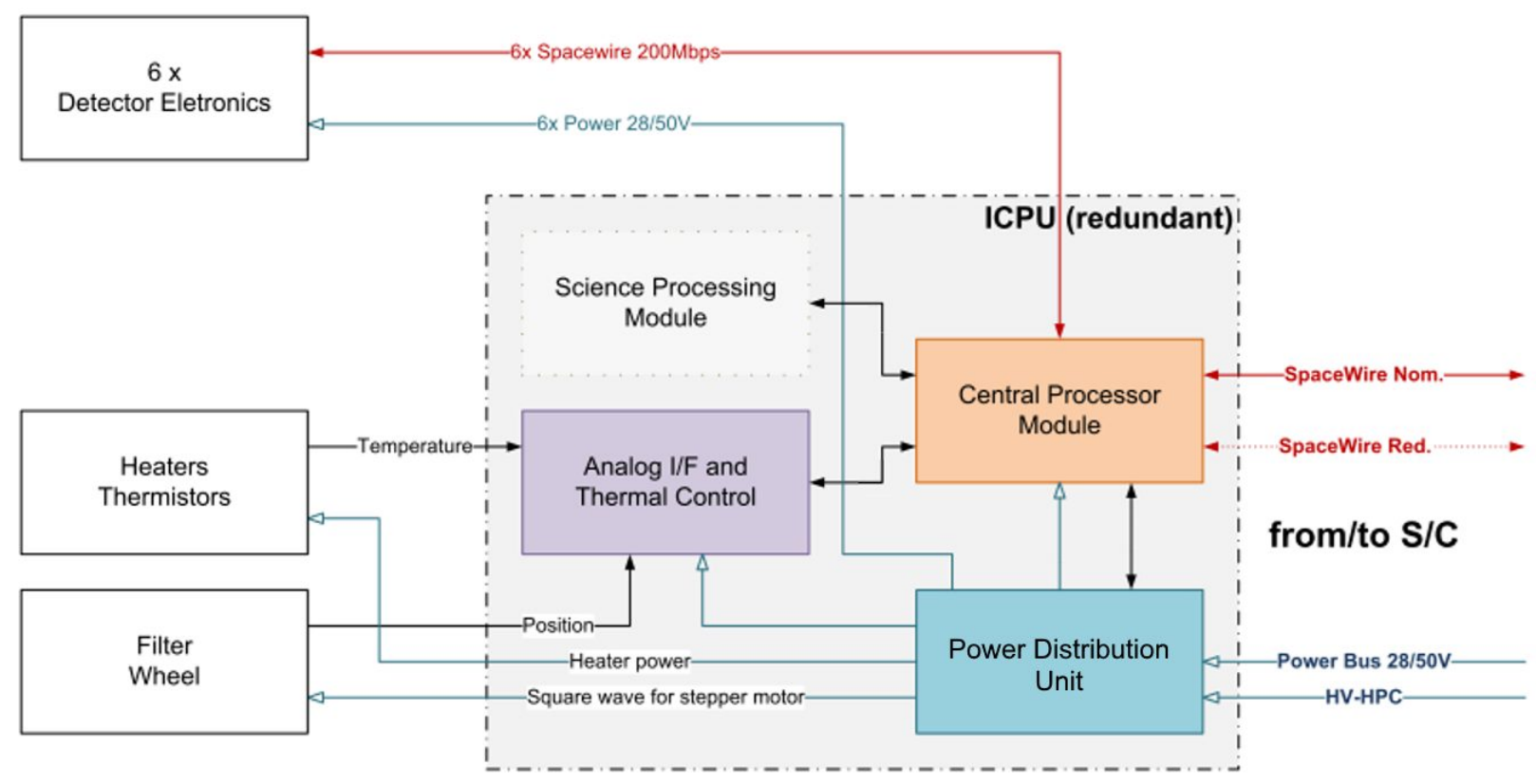

Figure 5. Instrument Control and Power-distribution Unit.

The ICPU comprises three (optionally four) modules:

- The Central Processor Module includes the WFI instrument controller. This module interfaces to all six DEs. The scientific data streams from the DEs are merged, compressed and sent to the mass memory of the satellite for downlink storage. SpaceWire communication lines are baselined between ICPU and spacecraft as well as between DEs and ICPU.

- The Power Distribution Unit (WFI secondary power supply) distributes the power received from the spacecraft to all other WFI subsystems. Latch relays and latch current limiters (tbc) are included to allow power on and off of WFI by high power commanding (HPC) of the spacecraft. Furthermore, the FW controller is placed on this module.

- The Analog I/F Board is a mixed signal board that collects all housekeeping data (including FW position detection) and performs temperature control of the detectors inside the $\mathrm{CH}$ and the DEs (tbc).

Optionally, a Science Products Module can be implemented in the ICPU dedicated to perform onboard data analysis if necessary.

\section{WFI LABORATORY ELECTRONICS AND BREADBOARDING}

\subsection{Laboratory DAQ System}

The WFI laboratory electronics is being developed in order to operate newly developed DEPFET detectors. DEPFET sensors in various technology options are designed and manufactured by Max-Planck semiconductor laboratory HLL. The principle architecture of this DAQ (Data Acquisition) electronics (see Figure 6) is based on WFI flight model design. FPGA-based detector control and read-out is implemented which will allow reuse of HDL (Hardware Description Language) code developed for FPGA configuration. 


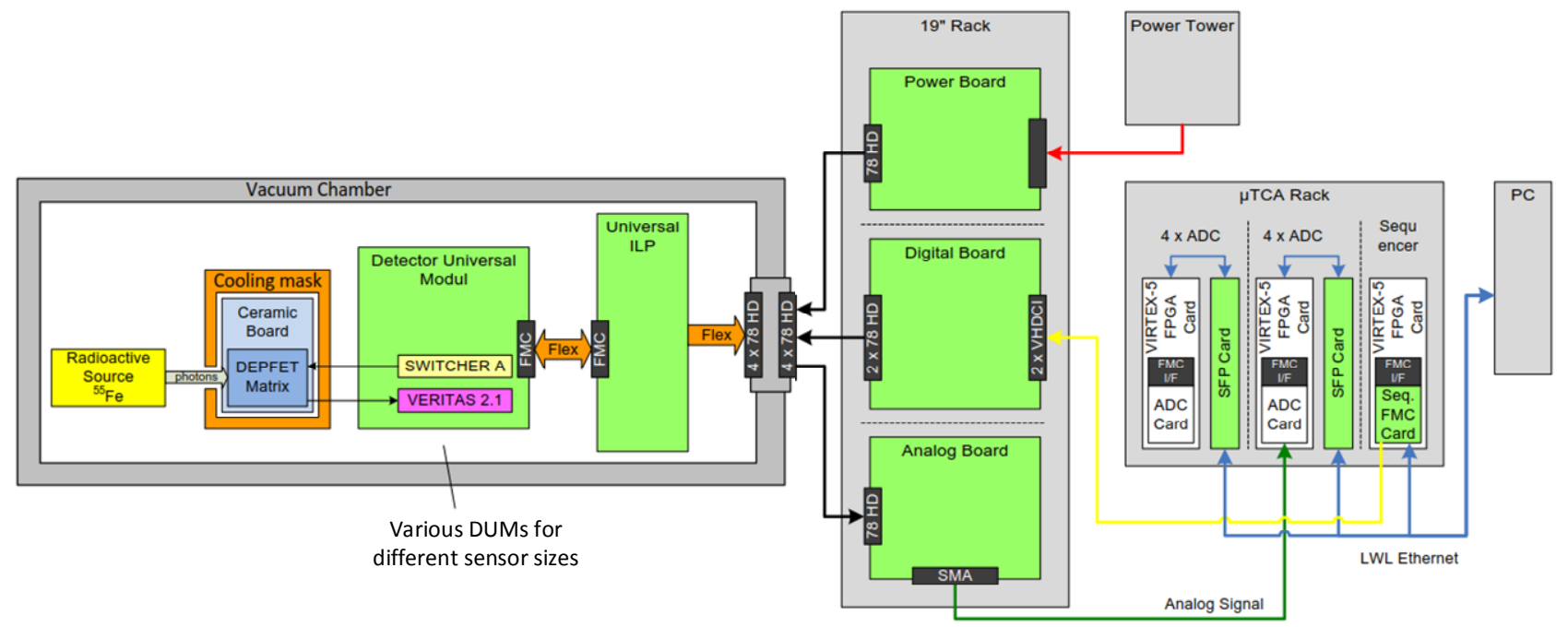

Figure 6. WFI laboratory DAQ system block diagram.

Inside a vacuum chamber a radioactive ${ }^{55} \mathrm{Fe}$ source generates X-ray photons that are detected by the DEPFET sensor. The sensors are glued to a ceramic support structure which is thermally controlled at a temperature of approximately $-70^{\circ} \mathrm{C}$. The ceramic board is connected to a suitable Detector Universal Module, a printed circuit board (PCB) which contains the Switcher-A and Veritas 2.1 ASICs. The universal ILP is a mother board for the Detector Universal Module that provides FPGA-based routing of all signals as well as buffering and energy-storage capacitances for the detector.

Figure 7 shows the DE laboratory system and the test setup to be used inside the vacuum chamber.
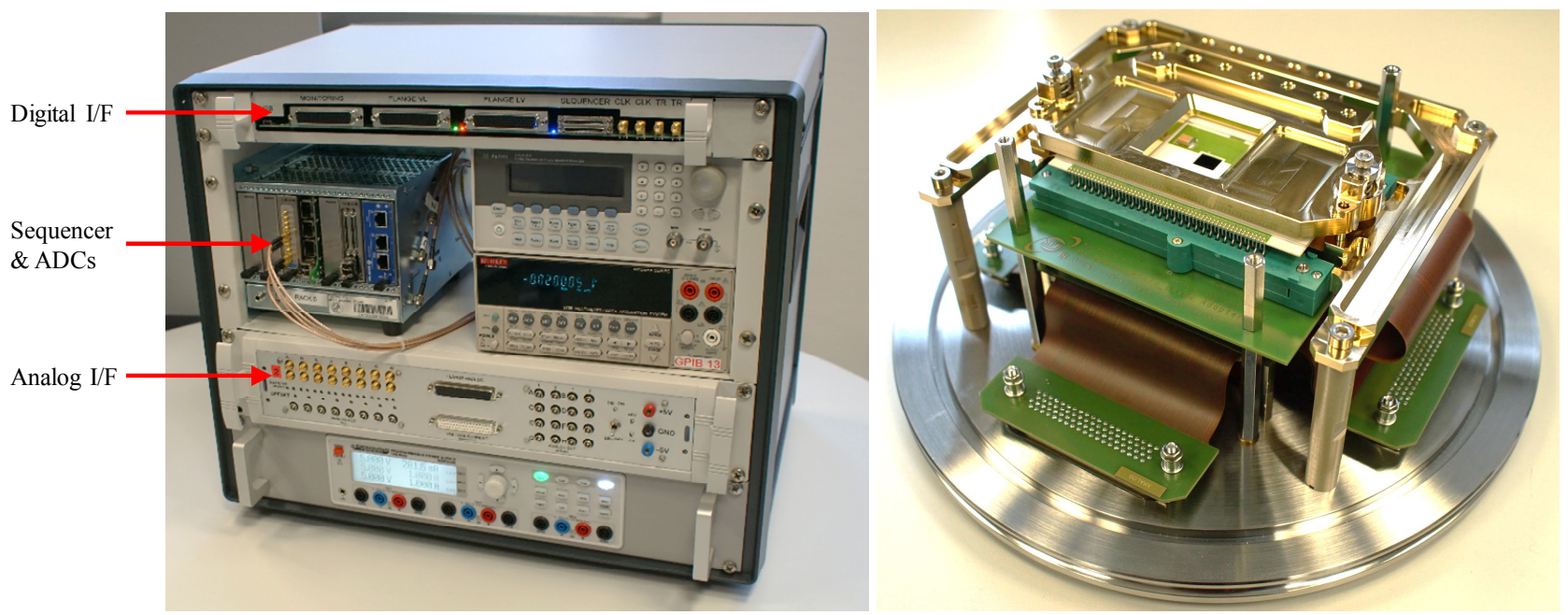

Figure 7. Left: DE laboratory system. Right: Vacuum flange with universal ILP and detector support incl. cooling mask.

The sequencer generating the digital control signals for the Switcher-A and the signal shaping and multiplexing sequence for the Veritas-2 ASICs is programmed on a commercial VIRTEX-5 FPGA card in a $\mu$ TCA Rack System. These signals are routed via the digital interface board to the vacuum chamber. The analog readout signals from the Veritas ASICs are buffered and filtered via the analog board and digitized by an analog-to-digital converter (ADC). All FPGA cards in the $\mu$ TCA rack are connected through Analog I/F optical fiber Ethernet to a host PC which serves as user interface to control and program the setup. 
The WFI laboratory system is already in use and shows good results with currently tested prototype detectors. Further development of the electronic system ensures test and operation of all newly developed DEPFET sensors and FEE particularly with regard to implementation into WFI flight model.

\subsection{WFI Detector Emulator}

In order to optimize DE performance, a Programmable Real-time Emulator (PRE) is being developed that shall be used for end-to-end WFI instrument verification and FP algorithm optimization (see also section 2.2).

In laboratory environment, the signal processing chain of the WFI breadboard components is analyzed with signals from given X-ray sources. It is not feasible to generate all required combinations of fast precise timing, high spatial resolution and all possible energy levels. To overcome this issue, a new end-to-end evaluation method has been introduced [4]. Complementary to the signal processing chain depicted in Figure 3, the DEPFET detector is substituted by a PRE as illustrated in Figure 8.

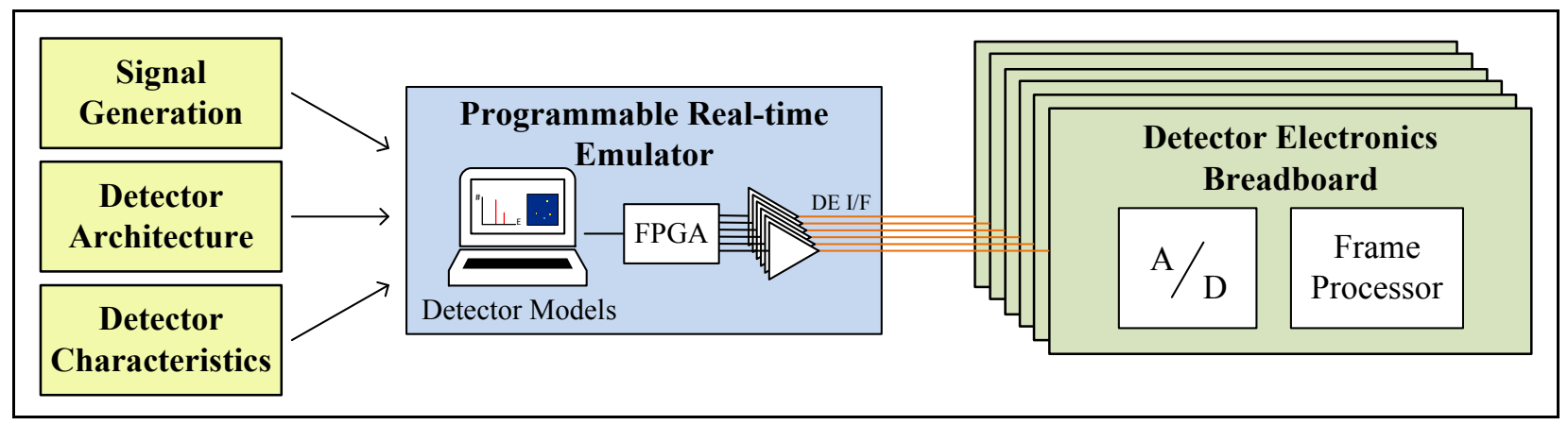

Figure 8. The Programmable Real-time Emulator (PRE) combined with the Detector Electronics.

Connecting the PRE with the DE allows to fully control the simulated WFI observation scenario. The input can be defined without the need of using X-ray sources.

Specific models of the detector were developed which can be interactively controlled and varied. A comparison of the DE digital output data after frame processing with the well-known input data is now feasible. The detector models describe the physical characteristics and the behavior of the sensor including its front-end electronics. The model parameters can be adjusted to set different detector and input characteristics. Reproducible measurements with constant conditions can be achieved.

The model Signal Generation comprises the emulation of X-ray photons with a particular energy, spatial distribution and hit time. With different parameters of the Detector Architecture it is possible to define pixel type, number of pixels and readout mode. The block Detector Characteristics implies models to emulate timing effects like misfit generation, pileup or influences of rolling shutter mode and readout speed. Each pixel can be modeled with varying offset, commonmode and gain per readout channel. Influences of noise can be analyzed by adding thermal, shot and 1/f noise to the signal. Figure 9 shows the PRE in its current state.

First results of an observed ${ }^{55} \mathrm{Fe}$ source show a good agreement of the simulated and measured spectra. Extensive testing of WFI Detector Electronics using PRE input will allow for optimization of FP algorithms. 


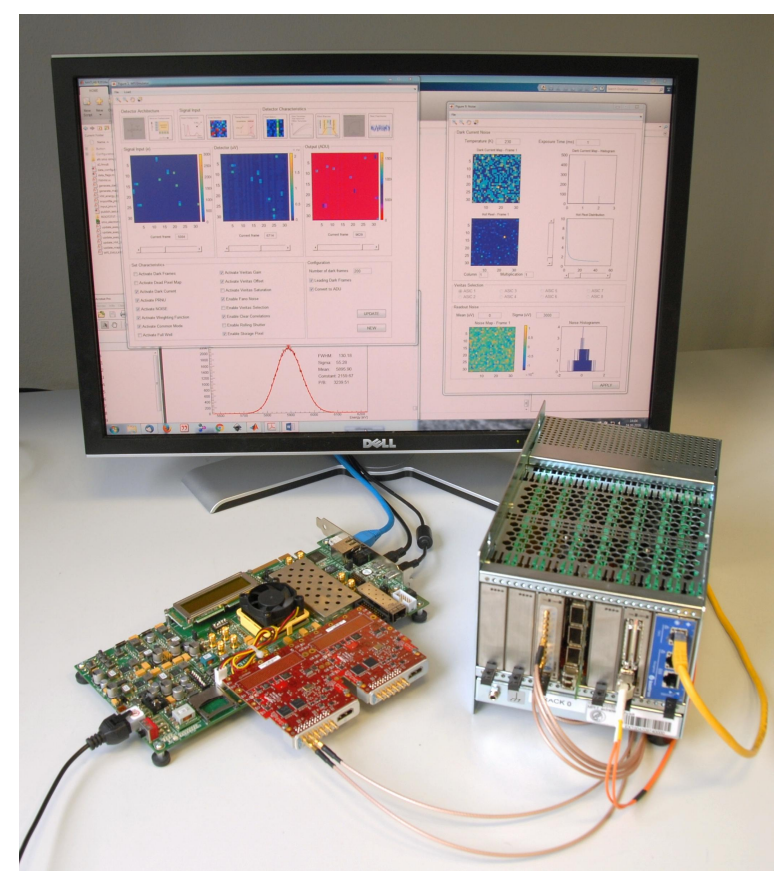

Figure 9. PRE setup combined with laboratory DE.

\section{CONCLUSION AND OUTLOOK}

The WFI technology development activity has been started. Conceptual design of the WFI electronics has been introduced and challenging areas like real-time data processing have been discussed. As soon as all technologies that are required for WFI with regard to electronics and data processing (DEPFET sensors, front-end electronics and frame processor electronics) are defined and selected, a WFI breadboard model will be developed in order to verify basic functionality. The PRE setup will be used for optimization of FP algorithms.

\section{ACKNOWLEDGMENTS}

The work was partly funded by the German space agency DLR (FKZ: 50QR1501 and 50QR1401) and the Austrian Research Promotion Agency (FFG) in the framework of the 11 Austrian Space Applications Program (ASAP 847987).

\section{REFERENCES}

[1] Rau, A., “Athena Wide Field Imager Key Science Drivers," Proceedings of SPIE 9905-79, Astronomical Telescopes and Instrumentation, (2016).

[2] Rogers, D., et al., "Athena Environmental Specification," TEC-EES/2014.89/DR, ESA Estec, (2014).

[3] Meidinger, N., "The Wide-Field Imager Instrument for Athena," Proceedings of SPIE 9905-78, Astronomical Telescopes and Instrumentation, (2016).

[4] Ott, S. et al., "New evaluation concept of the Athena WFI camera system by emulation of X-ray DEPFET detectors," Journal of Instrumentation, Vol. 11, (2016) 